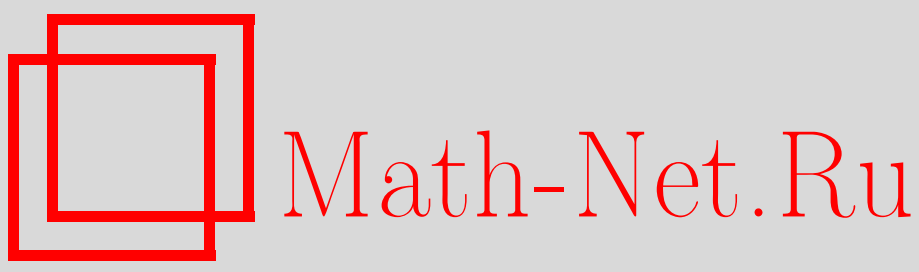

M. Sh. Shabozov, M. R. Langarshoev, Best linear approximation methods for some classes of analytic functions on the unit disk, Sibirsk. Mat. Zh., 2019, Volume 60, Number 6, 1414-1423

DOI: https://doi.org/10.33048/smzh.2019.60.618

Use of the all-Russian mathematical portal Math-Net.Ru implies that you have read and agreed to these terms of use http://www . mathnet.ru/eng/agreement

Download details:

IP: 52.6 .47 .48

April 26, 2023, 13:56:15

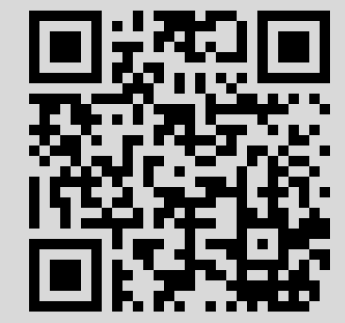


Сибирский математический журнал

Ноябрь-декабрь, 2019. Том 60, № 6

УДК 517.5

\title{
О НАИЛУЧШИХ ЛИНЕЙНЫХ МЕТОДАХ ПРИБЛИЖЕНИЯ НЕКОТОРЫХ КЛАССОВ АНАЛИТИЧЕСКИХ В ЕДИНИЧНОМ КРУГЕ ФУНКЦИЙ
}

М. Ш. Шабозов, М. Р. Лангаршоев

\begin{abstract}
Аннотация. В банаховых пространствах Харди и весовом пространстве Бергмана найдены точные значения бернштейновских, колмогоровских, гельфандовских и линейных $n$-поперечников классов аналитических в единичном круге функций, у которых усредненные с весом модули непрерывности $r$-х производных мажорируются заданной функцией, удовлетворяющей некоторым ограничениям.
\end{abstract}

DOI $10.33048 /$ smzh.2019.60.618

Ключевые слова: наилучшие линейные методы приближения, модуль непрерывности, весовое пространства Бергмана, мажоранта, $n$-поперечники, наилучший метод кодирования.

1. Пусть $X$ - произвольное банахово пространство, $S$ - единичный шар в $X, \Lambda_{n} \subset X$ - произвольное подпространство размерности $n, \Lambda^{n} \subset X-$ линейное подпространство коразмерности $n, \mathscr{L}\left(f, \Lambda_{n}\right)$ - линейный непрерывный оператор, переводящий $X$ в $\Lambda_{n}$. Через $E\left(f, \Lambda_{n}\right)_{X}$ обозначим наилучшее приближение функции $f \in X$ элементами $\varphi \in \Lambda_{n}$ :

$$
E\left(f, \Lambda_{n}\right)_{X}=\inf \left\{\|f-\varphi\|_{X}: \varphi \in \Lambda_{n}\right\},
$$

а через

$$
\mathscr{E}\left(f, \mathscr{L}\left(f, \Lambda_{n}\right)\right)_{X}=\left\|f-\mathscr{L}\left(f, \Lambda_{n}\right)\right\|_{X}
$$

- уклонение функции $f \in X$ от линейного непрерывного оператора $\mathscr{L}\left(f, \Lambda_{n}\right)$ в метрике пространства $X$. Для центрально-симметричного множества $\mathscr{M} \subset X$ полагаем

$$
\begin{gathered}
E\left(\mathscr{M}, \Lambda_{n}\right)_{X} \stackrel{\text { def }}{=} \sup \left\{E\left(f, \Lambda_{n}\right)_{X}: f \in \mathscr{M}\right\}, \\
\mathscr{E}\left(\mathscr{M}, \mathscr{L}, \Lambda_{n}\right)_{X} \stackrel{\text { def }}{=} \sup \left\{\mathscr{E}\left(f, \mathscr{L}\left(f, \Lambda_{n}\right)\right)_{X}: f \in \mathscr{M}\right\} .
\end{gathered}
$$

Величины

$$
\begin{gathered}
b_{n}(\mathscr{M} ; X)=\sup \left\{\sup \left\{\varepsilon>0:\left(\varepsilon S \cap \Lambda_{n+1}\right) \subset \mathscr{M}\right\}: \Lambda_{n+1} \subset X\right\}, \\
d^{n}(\mathscr{M} ; X)=\inf \left\{\sup \left\{\|f\|_{X}: f \in \mathscr{M} \cap \Lambda^{n}\right\}: \Lambda^{n} \subset X\right\}, \\
d_{n}(\mathscr{M} ; X)=\inf \left\{E\left(\mathscr{M}, \Lambda_{n}\right)_{X}: \Lambda_{n} \in X\right\}, \\
\delta_{n}(\mathscr{M} ; X)=\inf \left\{\inf \left\{\mathscr{E}\left(\mathscr{M}, \mathscr{L}, \Lambda_{n}\right)_{X}: \mathscr{L}: X \rightarrow \Lambda_{n}\right\}: \Lambda_{n} \subset X\right\}
\end{gathered}
$$

(с) 2019 Шабозов М. Ш., Лангаршоев М. Р. 
соответственно называют бернштейновским, гельфандовским, колмогоровским, линейным n-поперечниками. Между указанными величинами выполняются соотношения $[1,2]$

$$
b_{n}(\mathscr{M} ; X) \leq \frac{d_{n}(\mathscr{M} ; X)}{d^{n}(\mathscr{M} ; X)} \leq \delta_{n}(\mathscr{M} ; X) .
$$

2. В пространствах Харди $H_{q}, q \geq 1$, и Бергмана $\mathscr{B}_{q, \gamma}, q \geq 1$, с весом $\gamma \geq 0$ вопросы вычисления точных значений различных $n$-поперечников некоторых классов аналитических в единичном круге функций и построения наилучших линейных методов приближения рассматривались, например, в монографиях $[1,2]$ и работах [3-20]. Продолжим исследование в этом направлении и вычислим точные значения всех перечисленных выше $n$-поперечников классов аналитических в единичном круге функций $W_{a}^{(r)} X(\Phi, \mu), r \in \mathbb{N}, \mu \geq 1$ ( $X-$ пространство $H_{q}$ либо весовое пространство Бергмана $\left.\mathscr{B}_{q, \gamma}\right)$, у которых усредненные с весом модули непрерывности $r$-х производных по аргументу мажорируются заданной функцией $\Phi$, удовлетворяющей некоторым определенным естественным ограничениям.

Пусть $\mathbb{N}, \mathbb{R}_{+}, \mathbb{C}$ - множества натуральных, положительных и комплексных чисел соответственно, $U_{\rho}:=\{z \in \mathbb{C}:|z|<\rho\}-$ круг радиуса $\rho(0<\rho \leq 1)$, $U_{1}=U, A\left(U_{\rho}\right)$ - множество аналитических в круге $U_{\rho}$ функций.

Для произвольной $f \in A\left(U_{\rho}\right)$ положим

$$
M_{q}(f ; \rho) \stackrel{\text { def }}{=}\left(\frac{1}{2 \pi} \int_{0}^{2 \pi}\left|f\left(\rho e^{i t}\right)\right|^{q} d t\right)^{1 / q}, \quad 1 \leq q \leq \infty,
$$

где интеграл понимается в смысле Лебега. При $q=\infty$ будем предполагать функцию $f(z)$ непрерывной в замкнутом круге $\bar{U}_{\rho}:=\{z \in \mathbb{C}:|z| \leq \rho\}$. Символом $H_{q}, 1 \leq q \leq \infty$, обозначим банахово пространство Харди, состоящее из функций $f \in A(U)$, для которых конечна норма

$$
\|f\|_{q}:=\|f\|_{H_{q}}=\lim _{\rho \rightarrow 1-0} M_{q}(f ; \rho) .
$$

Известно, что норма (6) реализуется на угловых граничных значениях $f(t):=f\left(e^{i t}\right)$ функций $f \in H_{q}$. Символом $H_{q, \rho}, 1 \leq q \leq \infty, 0<\rho \leq 1$, $H_{q, 1} \equiv H_{q}$, обозначим пространство Харди функций $f \in A\left(U_{\rho}\right)$, для которых $\|f(z)\|_{q, \rho} \stackrel{\text { def }}{=}\|f(\rho z)\|_{q}<\infty$. Для любого $r \in \mathbb{N}$ через $f_{a}^{(r)}(z)$ обозначим производную $r$-го порядка функции $f \in A(U)$ по аргументу комплексного переменного $z=\rho \exp (i t)$. При этом

$$
f_{a}^{(1)}(z):=\frac{\partial f(z)}{\partial t}=\frac{d f(z)}{d z} \cdot \frac{\partial z}{\partial t}=f^{\prime}(z) z i \text { и } f_{a}^{(r)}(z)=\left\{f_{a}^{(r-1)}(z)\right\}_{a}^{(1)}, r \geq 2 .
$$

Под $H_{q, a}^{(r)}$ будем понимать класс функций $f \in A(U)$, у которых $f_{a}^{(r)} \in H_{q}, q \geq 1$.

Через $l_{q} \stackrel{\text { def }}{=} l_{q}(U), 1 \leq q<\infty$, обозначим банахово пространство комплекснозначных в круге $U$ функций $f$, имеющих конечную норму

$$
\|f\|_{l_{q}}=\left(\frac{1}{2 \pi} \iint_{(U)}|f(z)|^{q} d x d y\right)^{1 / q}=\left(\frac{1}{2 \pi} \int_{0}^{1} \int_{0}^{2 \pi} \rho\left|f\left(\rho e^{i t}\right)\right|^{q} d \rho d t\right)^{1 / q},
$$

где интеграл понимается в смысле Лебега. 
Пусть $\gamma(|z|) \geq 0$ - некоторая измеримая не эквивалентная нулю функция, суммируемая на множестве $U$. Через $l_{q, \gamma} \stackrel{\text { def }}{=} l_{q}(U, \gamma), 1 \leq q<\infty$, обозначим множество комплекснозначных функций $f$, для которых $\gamma^{1 / q} f \in l_{q}(U),\|f\|_{l_{q, \gamma}}=$ $\left\|\gamma^{1 / q} f\right\|_{l_{q}}$. Под $\mathscr{B}_{q, \gamma} \stackrel{\text { def }}{=} \mathscr{B}_{q}(U, \gamma), 1 \leq q<\infty$, понимаем банахово пространство функций $f \in A(U)$ таких, что $f \in l_{q, \gamma}$. При этом

$$
\|f\|_{\mathscr{B}_{q, \gamma}}=\left(\int_{0}^{1} \rho \gamma(\rho) M_{q}^{q}(f, \rho) d \rho\right)^{1 / q} .
$$

В частном случае, когда $\gamma \equiv 1$, пространство $\mathscr{B}_{q}:=\mathscr{B}_{q, 1}$ является обычным пространством Бергмана. Символом $\mathscr{B}_{q, \gamma, \rho}\left(1 \leq q \leq \infty, 0<\rho \leq 1, \mathscr{B}_{q, \gamma, 1} \equiv\right.$ $\left.\mathscr{B}_{q, \gamma}\right)$ обозначим пространство функций $f \in A\left(U_{\rho}\right)$, для которых

$$
\|f(z)\|_{\mathscr{B}_{q, \gamma, \rho}} \stackrel{\text { def }}{=}\|f(\rho z)\|_{\mathscr{B}_{q, \gamma}}<\infty
$$

а под $\mathscr{B}_{q, \gamma, a}^{(r)}$ будем понимать класс функций $f \in A(U)$, у которых $f_{a}^{(r)} \in \mathscr{B}_{q, \gamma}$, $1 \leq q \leq \infty$. В [16] доказано, что по сравнению с $\mathscr{B}_{q}$ пространство $\mathscr{B}_{q, \gamma}$ позволяет изучать функции $f \in A(U)$ с менее жесткими ограничениями на их поведение вблизи граничной окружности $\Gamma:=\{\zeta \in \mathbb{C}:|\zeta|=1\}$. Очевидно, что $H_{q} \subset \mathscr{B}_{q} \subset \mathscr{B}_{q, \gamma}, 1 \leq q<\infty$. Под $X:=X(U)$ будем понимать любое из вышеприведенных банаховых пространств $H_{q}, \mathscr{B}_{q, \gamma}$, а $X_{\rho}:=X_{\rho}(U)$ означает $H_{q, \rho}$ или $\mathscr{B}_{q, \gamma, \rho}$. Аналогичным образом под $X_{a}^{(r)}:=X_{a}^{(r)}(U)$ будем понимать $H_{q, a}^{(r)}$ либо $\mathscr{B}_{q, \gamma, a}^{(r)}$, а под $X_{\rho, a}^{(r)}-H_{q, \rho, a}^{(r)}$ либо $\mathscr{B}_{q, \gamma, \rho, a}^{(r)}$.

Для произвольной функции $f \in X(U)$ запишем модуль непрерывности

$$
\omega(f ; 2 t)_{X}=\sup \left\{\left\|f\left(z e^{i h}\right)-f\left(z e^{-i h}\right)\right\|_{X}:|h| \leq t\right\} .
$$

Обозначим через $\mathscr{P}_{n}$ множество комплексных алгебраических полиномов

$$
p_{n}(z)=\sum_{k=0}^{n} a_{k} z^{k} \quad\left(n \in \mathbb{N}, a_{k} \in \mathbb{C}\right)
$$

степени $n$, а равенством

$$
E_{n}(f)_{X}=E\left(f, \mathscr{P}_{n}\right)_{X} \stackrel{\text { def }}{=} \inf \left\{\left\|f-p_{n}\right\|_{X}: p_{n} \in \mathscr{P}_{n}\right\}
$$

определим наилучшее приближение функции $f \in X(U)$ элементами $\mathscr{P}_{n}$.

Пусть $\Phi(u)$ - положительная неубывающая функция, определенная для $u \geq 0$ и удовлетворяющая условию $\lim \{\Phi(u): u \rightarrow 0\}=\Phi(0)=0$. Используя функцию $\Phi$ в качестве мажоранты, для произвольных $\mu \geq 1$ и любых $r \in \mathbb{N}$ введем в рассмотрение класс функций

$$
\begin{aligned}
& W_{a}^{(r)} X(\Phi, \mu) \\
& =\left\{f \in X_{a}^{(r)}: \frac{1}{h} \int_{0}^{h} \omega\left(f_{a}^{(r)} ; 2 t\right)_{X}\left[1+\left(\mu^{2}-1\right) \sin \frac{\pi t}{2 h}\right] d t \leq \Phi(h), h \in(0, \pi]\right\} .
\end{aligned}
$$

В $[18,19]$ соответственно при $X=H_{q}$ и $X=\mathscr{B}_{q, \gamma}$ доказано, что если при заданном $\mu \geq 1$ и любых $h \in(0, \pi], n \in \mathbb{N}$, мажоранта $\Phi$ удовлетворяет условию

$$
\frac{\Phi(h)}{\Phi(\pi /(2 \mu n))} \geq \frac{\pi}{2 \mu} \int_{0}^{1}(\sin n h t)_{*}\left[1+\left(\mu^{2}-1\right) \sin \frac{\pi t}{2}\right] d t
$$


где

$$
(\sin u)_{*}= \begin{cases}\sin u, & \text { если } 0<u \leq \pi / 2, \\ 1, & \text { если } u>\pi / 2\end{cases}
$$

то при любых $n, r \in \mathbb{N}$ справедливы равенства

$$
\begin{aligned}
b_{n}\left(W_{a}^{(r)} X(\Phi ; \mu) ; X(U)\right)=d_{n} & \left(W_{a}^{(r)} X(\Phi ; \mu) ; X(U)\right) \\
& =E_{n-1}\left(W_{a}^{(r)} X(\Phi ; \mu)\right)_{X(U)}=\frac{\pi}{4 \mu n^{r}} \Phi\left(\frac{\pi}{2 \mu n}\right) .
\end{aligned}
$$

При этом условие (7) выполняется, например, для мажоранты $\Phi_{*}(h)=h^{\alpha}$, где

$$
\alpha:=\alpha(\mu)=\left(\frac{\pi}{2 \mu}\right)^{2} \int_{0}^{1} t \cos \left(\frac{\pi t}{2 \mu}\right)\left[1+\left(\mu^{2}-1\right) \sin \left(\frac{\pi t}{2}\right)\right] d t .
$$

Из равенства (9) вытекает, что $\alpha(1)=(\pi / 2)-1, \lim \{\alpha(\mu): \mu \rightarrow \infty\}=1$ и при всех значениях $\mu \in[1, \infty)$ имеет место неравенство $(\pi / 2)-1 \leq \alpha(\mu) \leq 1$.

Так как $X \subset X_{\rho}(0<\rho \leq 1)$, представляет несомненный интерес распространить равенство (8) с перечисленных выше $n$-поперечников (1)-(4) на более общее пространство $X_{\rho}(0<\rho \leq 1)$ :

$$
\begin{aligned}
\lambda_{n}\left(W_{a}^{(r)} X(\Phi ; \mu)\right. & \left.: X_{\rho}(U)\right)=E_{n-1}\left(W_{a}^{(r)} X(\Phi ; \mu)\right)_{X_{\rho}(U)} \\
& =\mathscr{E}\left(W_{a}^{(r)} X(\Phi ; \mu), \mathscr{L}_{\rho, r-1}, \mathscr{P}_{n-1}\right)_{X_{\rho}(U)}=\frac{\pi \rho^{n}}{4 \mu n^{r}} \Phi\left(\frac{\pi}{2 \mu n}\right),
\end{aligned}
$$

где $\lambda_{n}(\cdot)$ - любой из $n$-поперечников $b_{n}(\cdot), d^{n}(\cdot), d_{n}(\cdot)$, либо $\delta_{n}(\cdot)$.

В самом деле, для всех $n$-поперечников равенство (10) в случае, когда $X$ есть $\mathscr{B}_{q, \gamma}$, следует из результата теорем 2.1 и 3.1 в [18], а в случае $X=H_{q}$, оно выводится практически аналогичным образом. При этом наилучший линейный метод, реализующий точное значение линейного $n$-поперечника, имеет вид

$$
\mathscr{L}_{\rho, r-1}\left(f, \mathscr{P}_{n} ; z\right)=c_{0}(f)+\sum_{k=1}^{n-1} \mu_{k, \rho, r-1} c_{k}(f) z^{k},
$$

где $c_{k}(f)$ - коэффициенты Тейлора функции $f$,

$$
\begin{gathered}
\mu_{k, \rho, r-1} \stackrel{\text { def }}{=} 1-\rho^{2(n-k)}\left(\frac{k}{2 n-k}\right)^{r-1}\left\{1-\gamma_{k, n}\left(1-\frac{k^{2}}{(2 n-k)^{2}}\right)\right\}, \quad k=\overline{1, n-1} \\
\gamma_{k, n} \stackrel{\text { def }}{=} n \mu \int_{0}^{\pi /(2 n)} \cos k x \cos n x d x, \quad k=\overline{1, n-1} .
\end{gathered}
$$

Следуя [13], символом $\widetilde{\mathscr{P}}_{n-1}$ обозначим $n$-мерное подпространство, порожденное базисом

$$
\begin{gathered}
\widetilde{\varphi}_{k}(z)=\left\{1-\left(\frac{k}{2 n-1}\right)^{r-1}\left[1-\gamma_{k, n}\left(1-\left(\frac{k}{2 n-1}\right)^{2}\right)\right]|z|^{2(n-k)}\right\} z^{k}, \\
k=0,1, \ldots, n-1, \quad r \in \mathbb{N} .
\end{gathered}
$$

Для произвольной функции $f \in X(U)$ полагаем

$$
\widetilde{\mathscr{L}}_{r-1}\left(f, \widetilde{\mathscr{P}}_{n-1} ; z\right)=\sum_{k=0}^{n-1} c_{k}(f) \widetilde{\varphi}_{k}(z) .
$$

В принятых обозначениях справедлива 
Теорема 1. Для любых $\mu \geq 1, r, n \in \mathbb{N}$ справедливы равенства

$$
\begin{aligned}
\lambda_{n}\left(W_{a}^{(r)} X(\Phi ; \mu) ; l_{q, \gamma}\right) & =\bar{\lambda}_{n}\left(W_{a}^{(r)} X(\Phi ; \mu) ; \mathscr{B}_{q, \gamma}\right) \\
& =\mathscr{E}\left(W_{a}^{(r)} X(\Phi ; \mu), \widetilde{\mathscr{L}}_{r-1}, \widetilde{\mathscr{P}}_{n-1}\right)_{\mathscr{B}_{q, \gamma}} \\
= & \frac{\pi}{4 \mu n^{r}} \Phi\left(\frac{\pi}{2 \mu n}\right)\left(\int_{0}^{1} \rho^{n q+1} \gamma(\rho) d \rho\right)^{1 / q}, \quad 1 \leq q \leq \infty,
\end{aligned}
$$

где $\lambda_{n}(\cdot)$ - любой из $n$-поперечников $b_{n}(\cdot), d^{n}(\cdot), d_{n}(\cdot), \delta_{n}(\cdot)$, а $\bar{\lambda}_{n}(\cdot)-$ один из $n$-поперечников $d^{n}(\cdot)$ либо $b_{n}(\cdot)$.

ДокАзАТЕЛЬСтво. В самом деле, повторяя буквально схему рассуждений доказательства теоремы 2 в [13], убедимся, что для произвольной функции $f \in$ $W^{(r)} H_{q}(\Phi ; \mu)$ имеет место неравенство

$$
M_{q}\left(f-\widetilde{\mathscr{L}}_{r-1}\left(f, \widetilde{\mathscr{P}}_{n-1}\right) ; \rho\right) \leq \frac{\pi \rho^{n}}{4 \mu n^{r}} \Phi\left(\frac{\pi}{2 \mu n}\right) .
$$

Возведя обе части неравенства (13) в степень $q(1 \leq q \leq \infty)$, затем умножая на $\rho \gamma(\rho)$ и интегрируя по $\rho$ в пределах от 0 до 1, с учетом определения пространства $\mathscr{B}_{q, \gamma}$ запишем

$$
\mathscr{E}\left(W_{a}^{(r)} X(\Phi ; \mu), \widetilde{\mathscr{L}_{r-1}}, \widetilde{\mathscr{P}}_{n-1}\right)_{\mathscr{B}_{q, \gamma}} \leq \frac{\pi}{4 \mu n^{r}} \Phi\left(\frac{\pi}{2 \mu n}\right)\left(\int_{0}^{1} \rho^{n q+1} \gamma(\rho) d \rho\right)^{1 / q}
$$

Отсюда, учитывая определение линейного $n$-поперечника, получаем

$$
\delta_{n}\left(W_{a}^{(r)} X(\Phi ; \mu) ; \mathscr{B}_{q, \gamma}\right) \leq \frac{\pi}{4 \mu n^{r}} \Phi\left(\frac{\pi}{2 \mu n}\right)\left(\int_{0}^{1} \rho^{n q+1} \gamma(\rho) d \rho\right)^{1 / q} .
$$

Так как пространство $\mathscr{B}_{q, \gamma}$ изоморфно и изометрично вложено в пространство $l_{q, \gamma}$, на основании определений и свойств бернштейновского и гельфандовского $n$-поперечников [2, гл. II, $\S 3$, предложение 3.2$]$ получаем равенства

$$
\begin{aligned}
& d^{n}\left(W_{a}^{(r)} H_{q}(\Phi ; \mu) ; \mathscr{B}_{q, \gamma}\right)=d^{n}\left(W_{a}^{(r)} H_{q}(\Phi ; \mu) ; l_{q, \gamma}\right), \\
& b_{n}\left(W_{a}^{(r)} H_{q}(\Phi ; \mu) ; \mathscr{B}_{q, \gamma}\right)=b_{n}\left(W_{a}^{(r)} H_{q}(\Phi ; \mu) ; l_{q, \gamma}\right) .
\end{aligned}
$$

Из неравенств (5), (15) и равенств (16) получаем оценки сверху всех рассматриваемых $n$-поперечников. Для получения оценок снизу указанных $n$-поперечников введем в рассмотрение $(n+1)$-мерный шар полиномов

$$
\widetilde{S}_{n+1}:=\left\{p_{n} \in \mathscr{P}_{n}:\left\|p_{n}\right\|_{\mathscr{B}_{q, \gamma}} \leq \frac{\pi}{4 \mu n^{r}} \Phi\left(\frac{\pi}{2 \mu n}\right)\left(\int_{0}^{1} \rho^{n q+1} \gamma(\rho) d \rho\right)^{1 / q}\right\}
$$

и докажем включение $\widetilde{S}_{n+1} \subset W_{a}^{(r)} H_{q}(\Phi ; \mu)$. Отметим, что для произвольного полинома $p_{n} \in \mathscr{P}_{n}$ имеет место неравенство

$$
\omega\left(\left(p_{n}\right)_{a}^{(r)} ; 2 t\right)_{X} \leq 2 n^{r}(\sin n t)_{*}\left\|p_{n}\right\|_{X}
$$


доказательство которого при $X=H_{q}$ имеется в [4], а при $X=\mathscr{B}_{q, \gamma}-$ в [18]. В [16] доказано, что для любого полинома $p_{n} \in \mathscr{P}_{n}$ при всех $1 \leq q \leq \infty$ и любых $r, n \in \mathbb{N}$ имеет место неравенство

$$
\left\|\left(p_{n}\right)_{a}^{(r)}\right\|_{H_{q}} \leq n^{r}\left(\int_{0}^{1} \rho^{n q+1} \gamma(\rho) d \rho\right)^{-1 / q}\left\|p_{n}\right\|_{\mathscr{B}_{q, \gamma}} .
$$

Из неравенства (17), учитывая (18), получаем

$$
\omega\left(\left(p_{n}\right)_{a}^{(r)} ; 2 t\right)_{H_{q}} \leq 2 n^{r}(\sin n t)_{*}\left(\int_{0}^{1} \rho^{n q+1} \gamma(\rho) d \rho\right)^{-1 / q}\left\|p_{n}\right\|_{\mathscr{B}_{q, \gamma}} .
$$

Используя неравенство (19) для произвольной $p_{n} \in \widetilde{S}_{n+1}$, с учетом ограничения (7) будем иметь

$$
\begin{aligned}
& \frac{1}{h} \int_{0}^{h} \omega\left(\left(p_{n}\right)_{a}^{(r)} ; 2 t\right)_{H_{q}}\left[1+\left(\mu^{2}-1\right) \sin \frac{\pi t}{2 h}\right] d t \\
& \leq 2 n^{r}\left(\int_{0}^{1} \rho^{n q+1} \gamma(\rho) d \rho\right)^{-1 / q}\left\|p_{n}\right\|_{\mathscr{B}_{q, \gamma}} \cdot \frac{1}{h} \int_{0}^{h}(\sin n t)_{*}\left[1+\left(\mu^{2}-1\right) \sin \frac{\pi t}{2 h}\right] d t \\
& \leq \frac{\pi}{2 \mu} \Phi\left(\frac{\pi}{2 \mu n}\right) \int_{0}^{1}(\sin n h t)_{*}\left[1+\left(\mu^{2}-1\right) \sin \frac{\pi t}{2}\right] d t \leq \Phi(h),
\end{aligned}
$$

откуда сразу следует включение $\widetilde{S}_{n+1} \subset W_{a}^{(r)} H_{q}(\Phi ; \mu)$. Из доказанного включения и определения бернштейновского $n$-поперечника получаем оценку снизу:

$$
\begin{aligned}
b_{n}\left(W_{a}^{(r)} H_{q}(\Phi ; \mu) ; \mathscr{B}_{q, \gamma}\right) \geq b_{n}\left(\widetilde{S}_{n+1} ; \mathscr{B}_{q, \gamma}\right) & \\
& \geq \frac{\pi}{4 \mu n^{r}} \Phi\left(\frac{\pi}{2 \mu n}\right)\left(\int_{0}^{1} \rho^{n q+1} \gamma(\rho) d \rho\right)^{1 / q} .
\end{aligned}
$$

Требуемые равенства (12) следуют из сопоставления неравенств (15), равенств (16) и неравенств (20). Теорема 1 доказана.

В экстремальных задачах теории аппроксимации аналитических в круге функций определенный интерес представляет вычисление точных верхних граней модулей коэффициентов Тейлора (см., например, $[13,16])$ на различных классах аналитических функций. Приведем решение указанной задачи для рассматриваемых нами классов функций.

Теорема 2. Пусть $L_{n}(\mathscr{M}):=\sup \left\{\left|c_{n}(f)\right|: f \in \mathscr{M}\right\}$. Тогда при любых $n \in \mathbb{N}, r \in \mathbb{Z}_{+}$и $q \in[1, \infty]$ справедливы равенства

$$
\begin{gathered}
L_{n}\left(W_{a}^{(r)} H_{q}(\Phi ; \mu)\right)=\frac{\pi}{4 \mu n^{r}} \Phi\left(\frac{\pi}{2 \mu n}\right), \\
L_{n}\left(W_{a}^{(r)} \mathscr{B}_{q, \gamma}(\Phi ; \mu)\right)=\frac{\pi}{4 \mu n^{r}} \Phi\left(\frac{\pi}{2 \mu n}\right)\left(\int_{0}^{1} \rho^{n q+1} \gamma(\rho) d \rho\right)^{-1 / q} .
\end{gathered}
$$


ДокАЗАТЕЛЬСтво. В самом деле, для произвольной функции $f \in A(U)$ коэффициент Тейлора $c_{n}(f)$ представим в виде

$$
\begin{aligned}
c_{n}(f)=\frac{1}{2 \pi i} \int_{|\zeta|=\rho} f(\zeta) \zeta^{-n-1} d \zeta & \\
= & \frac{1}{2 \pi \rho^{n}} \int_{0}^{2 \pi}\left[f\left(\rho e^{i t}\right)-\mathscr{L}_{\rho, r-1}\left(f, \mathscr{P}_{n} ; \rho e^{i t}\right)\right] e^{-i n t} d t,
\end{aligned}
$$

где $\mathscr{L}_{\rho, n-1}\left(f, \mathscr{P}_{n} ; \rho e^{i t}\right)$ - линейный оператор, определенный равенством (11). Из (21) на основании неравенства Гёльдера и равенств (10) для произвольной функции $f \in W_{a}^{(r)} H_{q}(\Phi ; \mu)$ имеем

$$
\left|c_{n}(f)\right| \leq \rho^{-n} \mathscr{E}\left(f ; \mathscr{L}_{\rho, r-1}\left(f, \mathscr{P}_{n}\right)\right)_{H_{q, \rho}} \leq \frac{\pi}{4 \mu n^{r}} \Phi\left(\frac{\pi}{2 \mu n}\right)
$$

откуда сразу вытекает оценка сверху:

$$
L_{n}\left(W_{a}^{(r)} H_{q}(\Phi ; \mu)\right) \leq \frac{\pi}{4 \mu n^{r}} \Phi\left(\frac{\pi}{2 \mu n}\right) .
$$

С другой стороны, для любых $\rho, R \in(0,1)$, записав коэффициент $c_{n}(f)$ в виде

$$
c_{n}(f)=\frac{1}{2 \pi(\rho R)^{n}} \int_{0}^{2 \pi}\left[f\left(\rho R e^{i \tau}\right)-\mathscr{L}_{\rho, r-1}\left(f, \mathscr{P}_{n} ; \rho R e^{i \tau}\right)\right] e^{-i n \tau} d \tau,
$$

в силу неравенства Гёльдера запишем

$$
R^{n}\left|c_{n}(f)\right| \leq \rho^{-n} M_{q}\left(f-\mathscr{L}_{\rho, r-1}\left(f, \mathscr{P}_{n}\right) ; \rho R\right),
$$

откуда с учетом определения нормы в пространствах $X(U)$ для произвольной функции $f \in W_{a}^{(r)} X(\Phi ; \mu)$ имеем

$$
\left|c_{n}(f)\right| \leq \rho^{-n} \mathscr{E}\left(f ; \mathscr{L}_{\rho, r-1}\left(f, \mathscr{P}_{n}\right) ; X_{\rho}\right)\left(\int_{0}^{1} \rho^{n q+1} \gamma(\rho) d \rho\right)^{-1 / q}
$$

Из этого неравенства и соотношений (10) получаем оценки сверху:

$$
\begin{aligned}
& L_{n}\left(W_{a}^{(r)} X(\Phi ; \mu)\right) \\
& \leq \frac{\pi}{4 \mu n^{r}} \Phi\left(\frac{\pi}{2 \mu n}\right) \begin{cases}1, & \text { если } X(U)=H_{q}, \\
\left(\int_{0}^{1} \rho^{n q+1} \gamma(\rho) d \rho\right)^{-1}, & \text { если } X(U)=\mathscr{B}_{q, \gamma} .\end{cases}
\end{aligned}
$$

Для получения оценки снизу рассмотрим функцию

$$
f_{0}(z)=\frac{1}{(i n)^{r}} \cdot \frac{\pi}{4 \mu} \Phi\left(\frac{\pi}{2 \mu n}\right) \frac{z^{n}}{\left\|z^{n}\right\|_{X}} \in W_{a}^{(r)} X(\Phi ; \mu) .
$$

Для этой функции согласно определению величины $L_{n}(\cdot)$ записываем оценки снизу:

$$
\begin{aligned}
& \mathscr{L}_{n}\left(W_{a}^{(r)} X(\Phi ; \mu)\right) \geq\left|c_{n}\left(f_{0}\right)\right| \\
&=\frac{\pi}{4 \mu n^{r}} \Phi\left(\frac{\pi}{2 \mu n}\right) \begin{cases}1, & \text { если } X(U)=H_{q}, \\
\left(\int_{0}^{1} \rho^{n q+1} \gamma(\rho) d \rho\right)^{-1}, & \text { если } X(U)=\mathscr{B}_{q, \gamma} .\end{cases}
\end{aligned}
$$


Равенства (21) и (22) получаем из сравнения оценок сверху (24) и снизу (25). Теорема 2 доказана.

3. В пп. 1, 2 вычислены точные значения различных поперечников указанных классов аналитических в единичном круге функций. С поперечниками рассматриваемых классов функций можно связать задачи оптимального восстановления и кодирования функций в интерпретации Н. П. Корнейчука $[21$, гл. $8, \S 8.3 ; 22]$.

Приведем необходимые понятия и определения. Пусть в нормированном функциональном пространстве $X$ задан набор $M_{n}:=\left\{\mu_{1}, \mu_{2}, \ldots, \mu_{n}\right\}$ определенных на $X$ функционалов $\mu_{k}, k=\overline{1, n}$. Множество $M_{n}$ можно рассматривать как метод кодирования, сопоставляющий функции $f \in X$ вектору $T\left(f, M_{n}\right)=$ $\left\{\mu_{1}(f), \ldots, \mu_{n}(f)\right\}$. Задачу восстановления функции $f$ по информации $T$ решают, сопоставляя вектору $T\left(f, M_{n}\right)$ функцию

$$
A\left(f, M_{n} ; G_{n}, \Gamma_{n}: z\right)=\sum_{k=1}^{n} \gamma_{k} \mu_{k}(f) g_{k}(z),
$$

где $G_{n}=\left\{g_{k}(z)\right\}_{k=1}^{n}$ и $\Gamma_{n}=\left\{\gamma_{k}\right\}_{k=1}^{n} \in \operatorname{Im}$ соответственно произвольные система линейно независимых функций из $X$ и набор числовых коэффициентов, позволяющий наилучшим образом воспроизводить элементы класса $\mathscr{M} \subset X$, $\operatorname{Im}=\left\{\Gamma_{n}\right\}-$ вектор числовых коэффициентов. Погрешность восстановления на классе $\mathscr{M}$ считают равной

$$
\mathscr{R}_{n}\left(\mathscr{M} ; M_{n}, G_{n}\right)=\inf \left\{\sup \left\{\left\|f-A\left(f ; M_{n}, G_{n}, \Gamma_{n}\right)\right\|_{B_{q, \gamma}}: f \in \mathscr{M}\right\}: \Gamma_{n} \subset C^{n}\right\}
$$

и полагают $\mathscr{R}_{n}(\mathscr{M}, X)=\inf \left\{\mathscr{R}\left(\mathscr{M} ; M_{n}, G_{n}\right): M_{n}, G_{n}\right\}$.

Пусть $M_{n}^{\prime}$ - набор заданных на $X$ линейных ограниченных функционалов. Тогда рассматривают следующую характеристику:

$$
\mathscr{R}_{n}^{\prime}(\mathscr{M}, X)=\inf \left\{\mathscr{R}\left(\mathscr{M} ; M_{n}^{\prime}, G_{n}\right): M_{n}^{\prime}, G_{n}\right\} .
$$

Метод восстановления $\left(\stackrel{\circ}{M_{n}}, \stackrel{\circ}{G_{n}}, \stackrel{\circ}{\Gamma_{n}}\right)\left\{\stackrel{\circ}{M_{n}^{\prime}}, \stackrel{\circ}{G_{n}}, \stackrel{\circ}{\Gamma_{n}}\right\}$, для которого

$$
\begin{aligned}
\mathscr{R}_{n}(\mathscr{M}, X) & =\sup \left\{\left\|f-A\left(f, \stackrel{\circ}{M}_{n}, \stackrel{\circ}{G_{n}}, \stackrel{\circ}{\Gamma_{n}}\right)\right\|_{X}: f \in \mathscr{M}\right\}, \\
\left\{\mathscr{R}_{n}^{\prime}(\mathscr{M}, X)\right. & \left.=\sup \left\{\left\|f-A\left(f, \stackrel{\circ}{M}_{n}^{\prime}, \stackrel{\circ}{G_{n}}, \stackrel{\circ}{\Gamma}_{n}\right)\right\|_{X}: f \in \mathscr{M}\right\}\right\},
\end{aligned}
$$

называют оптимальным (оптимальным линейным) методом восстановления функций из класса $\mathscr{M}$. Справедливы соотношения [21]

$$
\mathscr{R}_{n}^{\prime}(\mathscr{M}, X)=\lambda_{n}(\mathscr{M}, X), \quad \mathscr{R}_{n}(\mathscr{M}, X) \geq d_{n}(\mathscr{M}, X),
$$

причем если $\mathscr{M}=\widetilde{\mathscr{M}} \otimes L$, где $\widetilde{\mathscr{M}}$ - компакт, а $L-$ конечномерное подпространство, то в (27) везде имеет место равенство.

Наряду с (26) рассматривают также величину

$$
\mathscr{K}\left(\mathscr{M}, M_{n}\right)=\sup \left\{\left\|f_{1}-f_{2}\right\|_{X}: f_{1}, f_{2} \in \mathscr{M}, T\left(f_{1}, M_{n}\right)=T\left(f_{2}, M_{n}\right)\right\},
$$

которую можно интерпретировать как погрешность метода кодирования на классе $\mathscr{M}$ с помощью фиксированного набора функционалов $M_{n}$.

\section{Полагая}

$$
\nu^{n}(\mathscr{M}, X)=\inf \left\{\mathscr{K}\left(\mathscr{M}, M_{n}\right): M_{n}\right\},
$$

где нижняя грань берется по всем наборам $M_{n}$ линейных функционалов, определенных на сопряженном пространстве $X^{*}$, получаем

$$
\nu^{n}(\mathscr{M}, X) \leq 2 \mathscr{R}_{n}^{\prime}(\mathscr{M}, X),
$$

а если $\mathscr{M}$ - центрально-симметричное выпуклое множество, то

$$
\nu^{n}(\mathscr{M}, X)=2 d^{n}(\mathscr{M}, X) .
$$


Теорема 3. При выполнении условия (7) наилучший метод кодирования функций из класса $W_{a}^{(r)} X(\Phi ; \mu)$ в банаховом пространстве $X_{\rho}(U)$ доставляет набор $\stackrel{\circ}{M}_{n}$ линейных функционалов

$$
\stackrel{\circ}{\mu_{k}}(f)=c_{k}(f), \quad k=\overline{0, n-1} .
$$

Оптимальным линейным методом восстановления $\stackrel{\circ}{M_{n}^{\prime}}, \stackrel{\circ}{G_{n}}, \stackrel{\circ}{\Gamma_{n}}$ функций $f(z)$ класса $W_{a}^{(r)} X(\Phi ; \mu)$ в пространстве $X_{\rho}(U)$ является определенный равенством (11) линейный метод $\mathscr{L}_{\rho, r-1}\left(f, \mathscr{P}_{n-1} ; z\right)$. При этом для любого $n \in \mathbb{N}$

$$
\begin{aligned}
\nu^{n}\left(W_{a}^{(r)} X(\Phi ; \mu), X_{\rho}(U)\right)=\mathscr{R}_{n} & \left(W_{a}^{(r)} X(\Phi ; \mu), X_{\rho}(U)\right) \\
= & \mathscr{R}_{n}^{\prime}\left(W_{a}^{(r)} X(\Phi ; \mu), X_{\rho}(U)\right)=\frac{\pi \rho^{n}}{4 \mu n^{r}} \Phi\left(\frac{\pi}{2 \mu n}\right) .
\end{aligned}
$$

Теорема 4. При выполнении условия (7) оптимальным линейным методом восстановления элементов $f(z) \in W_{a}^{(r)} H_{q}(\Phi ; \mu)$ в банаховом пространстве $\mathscr{L}_{q, \gamma}$ является определенный в п. 2 линейный метод $\widetilde{V}_{r-1}\left(f, \widetilde{\mathscr{P}}_{n}, z\right)$, а наилучшим методом кодирования - набор функционалов (28). При этом для любого $n \in \mathbb{N}$ справедливы равенства

$$
\begin{aligned}
\frac{1}{2} \lambda^{n}\left(W_{a}^{(r)} H_{q}(\Phi ; \mu), \mathscr{L}_{q, \gamma}\right)=\mathscr{R}_{n}\left(W_{a}^{(r)} H_{q}(\Phi ; \mu), \mathscr{L}_{q, \gamma}\right) \\
=\mathscr{R}_{n}^{\prime}\left(W_{a}^{(r)} H_{q}(\Phi ; \mu), \mathscr{L}_{q, \gamma}\right)=\frac{\pi}{4 \mu n^{r}} \Phi\left(\frac{\pi}{2 \mu n}\right)\left(\int_{0}^{1} \rho^{n q+1} \gamma(\rho) d \rho\right)^{-1 / q} .
\end{aligned}
$$

Авторы благодарят рецензента за ценные замечания.

\section{ЛИТЕРАТУРА}

1. Тихомиров В. М. Поперечники множеств в функциональных пространствах и теория наилучших приближений // Успехи мат. наук. 1960. Т. 15, № 3. С. 81-120.

2. Pinkus A. $n$-Widths in approximation theory. Berlin: Springer-Verl., 1985.

3. Тайков Л. В. О наилучшем приближении в среднем некоторых классов аналитических функций // Мат. заметки. 1967. Т. 1, № 2. С. 155-162.

4. Тайков Л. В. Поперечники некоторых классов аналитических функций // Мат. заметки. 1977. T. 22, № 2. С. 285-294.

5. Айнуллоев Н., Тайков Л. В. Наилучшее приближение в смысле Колмогорова классов аналитических в единичном круге функций // Мат. заметки. 1986. Т. 40, № 3. С. 341-351.

6. Двейрин М. З. О приближении функций, аналитических в единичном круге // Метрические вопросы теории функций и отображений. Киев: Наук. думка, 1975. Т. 6. С. 41-54.

7. Фарков Ю. А. Поперечники классов Харди и Бергмана в шаре из $\mathbb{C}^{n} / /$ Успехи мат. наук. 1990. Т. 45, № 5. С. 197-198.

8. Farkov Yu. A. n-Widths, Faber expansion, and computation of analytic functions // J. Complexity. 1996. V. 2, N 1. P. 58-79.

9. Fisher S. D., Stessin M. I. The $n$-widths of the unit ball of $H^{q} / /$ J. Approx. Theory. 1991. V. 67, N 3. P. 347-356.

10. Вакарчук С. Б. Наилучшие линейные методы приближения и поперечники классов аналитических в круге функций // Мат. заметки. 1995. Т. 57, № 1. С. 30-39.

11. Вакарчук С. Б. О наилучших линейных методах приближения и поперечниках некоторых классов аналитических функций // Мат. заметки. 1999. Т. 65, № 2. С. 186-193.

12. Вакарчук С. Б. Точные значения поперечников классов аналитических в круге функций и наилучшие линейные методы // Мат. заметки. 2002. Т. 72, № 5. С. 665-669. 
13. Вакарчук С. Б. О некоторых экстремальных задачах теории приближений в комплексной плоскости // Укр. мат. журн. 2004. Т. 56, № 9. С. 1155-1171.

14. Вакарчук С. Б., Забутная В. И. О наилучших линейных методах приближения функций классов Л. В. Тайкова в пространствах Харди $H_{q, \rho}, q \geq 1,0<\rho \leq 1 / /$ Мат. заметки. 2009. Т. 85, № 3. С. 323-329.

15. Шабозов M. Ш., Лангаршоев M. Р. Наилучшее приближение некоторых классов функций в весовом пространстве Бергмана // Изв. АН Респ. Таджикистан. Отделение физ.мат., хим., геол. и тех. наук. 2009. Т. 136, № 3. С. 7-23.

16. Вакарчук С. Б., Шабозов М. Ш. О поперечниках классов функций, аналитических в круге // Мат. сб. 2010. Т. 201, № 8. С. 3-22.

17. Шабозов М. Ш., Лангаршоев М. Р. О наилучших линейных методах и значениях поперечников некоторых классов аналитических функций в весовом пространстве Бергмана // Докл. АН. 2013. Т. 450, № 5. С. 518-521.

18. Шабозов М. Ш., Саидусайнов М. С. Значения $n$-поперечников и наилучшие линейные методы приближения некоторых классов аналитических функций в весовом пространстве Бергмана // Изв. ТулГУ. Естественные науки. 2014. № 3. С. 40-57.

19. Шабозов М. Ш., Юсупов Г. А. Наилучшие линейные методы приближения и поперечники некоторых классов функций в пространстве Харди // Докл. АН Респ. Таджикистан. 2014. T. 57, № 2. C. 97-102.

20. Лангаршоев M. Р. О наилучших линейных методах приближения и точных значениях поперечников некоторых классов аналитических функций в весовом пространстве Бергмана // Укр. мат. журн. 2015. Т. 67, № 10. С. 1366-1379.

21. Корнейчук Н. П. Точные константы в теории приближения. М.: Наука, 1987.

22. Корнейчук $Н$. П. Поперечники в $L_{p}$ классов непрерывных и дифференцируемых функций и оптимальные методы кодирования и восстановления функций и их производных // Изв. АН СССР. Сер. мат. 1981. Т. 45, № 2. С. 266-290.

Поступила в редакцию 9 января 2017 г.

После доработки 31 мая 2019 г.

Принята к публикации 24 июля 2019 г.

Шабозов Мирганд Шабозович

Университет Центральной Азии,

ул. Дружбы народов, 47А, Душанбе 734013, Таджикистан;

Таджикский национальный университет,

пр. Рудаки, 17, Душанбе 734025, Таджикистан

shabozov@mail.ru

Лангаршоев Мухтор Рамазонович

Таджикский национальный университет,

пр. Рудаки, 17, Душанбе 734025, Таджикистан

mukhtor77@mail.ru 